\title{
Dissection of Level III Axillary Lymph Nodes in Breast Cancer
}

This article was published in the following Dove Press journal:

Cancer Management and Research

Jiejie $\mathrm{Hu}$

Xianghou Xia

Hongjian Yang

Yang Yu

Breast Surgery Department, Cancer Hospital of the University of Chinese Academy of Sciences (Zhejiang Cancer Hospital), Institute of Cancer and Basic Medicine (IBMC), Chinese Academy of Sciences, Hangzhou City, Zhejiang Province, People's Republic of China
Correspondence: Yang Yu

Breast Surgery Department, Cancer Hospital of the University of Chinese Academy of Sciences (Zhejiang Cancer Hospital), Institute of Cancer and Basic Medicine (IBMC), Chinese Academy of Sciences, No. I, Banshan East Road,

Gongshu District, Hangzhou City,

Zhejiang Province, 310022, People's

Republic of China

Tel $+86 \mid 375825268$ I

Email yuyangkaiyu@163.com
Abstract: Axillary lymph node dissection is an indispensable step in modified radical mastectomy for breast cancer. It is the most reliable method and the golden standard to determine the status of axillary lymph nodes. It is also of great importance to evaluate the prognosis and develop treatment plans for breast cancer patients. Axillary lymph node dissection can be anatomically divided into levels I, II, and III. Level I and Level II axillary lymph dissection is the standard clinical treatment of axillary lymph nodes positive breast cancer, whereas level III axillary lymph node dissection has been controversial. Level III axillary lymph node metastasis is one of the important factors that can easily cause distant metastasis and recurrence. It is also an important index to estimate the prognosis of breast cancer patients. To facilitate the decision of whether or not to perform level III lymph node dissection, we reviewed the indications, complications, and surgical procedures of level III lymph node dissection.

Keywords: breast cancer, axillary lymph node dissection, level III

\section{Introduction}

Axillary lymph node dissection is an indispensable step in modified radical mastectomy for breast cancer. It is the most reliable method and the golden standard to determine the status of axillary lymph nodes. It also is of great importance to evaluate the prognosis and develop treatment plans for breast cancer patients. Anatomically, axillary lymph nodes are divided into three levels, with the pectoralis minor muscle as the boundary. The lymph nodes located laterally to the pectoralis minor muscle are level I axillary nodes, which include the lateral breast group, the central group, and the subscapular group. The lymph nodes located posteriorly to the pectoralis minor muscle's deep surface are level II axillary lymph nodes. The lymph nodes located medially to the pectoralis minor muscle are level III axillary lymph nodes. Level III lymph nodes are the relay station of mammary gland lymphatic drainage to the supraclavicular lymph nodes or the thoracic duct. Level I and II axillary lymph dissection is the standard clinical treatment of axillary lymph nodes positive breast cancer, ${ }^{1}$ whereas level III axillary lymph node dissection has been controversial. To analyze whether level III lymph node dissection is necessary, we reviewed the indications, complications, and surgical procedures of level III lymph node dissection.

\section{Indications of Level III Axillary Lymph Node Dissection}

The NCCN Guidelines for clinical practice in breast cancer indicate that in the absence of gross disease in level II nodes, lymph node dissection should include tissue inferior to the axillary vein from the latissimus dorsi muscle laterally to the 
medial border of the pectoralis minor muscle (level I/II). ${ }^{2}$ Only in cases with gross diseases in level I/II, level III dissection to the thoracic inlet should be performed.

Dissection of level III may lead to postoperative paresthesia, axillary deformity, lymphedema, and so on. It is an irregular clinical procedure that has been controversial. Sentinel lymph node biopsy has a profound effect on the reduction of axillary trauma. The Z0011 trial clearly defined the lymph nodes positive breast cancer patients in whom it is safe to avoid axillary lymph node dissection: (1) with clinical T1 or T2 stage tumors; (2) with $\leq 2$ positive sentinel lymph nodes; (3) receiving breastconserving surgery; (4) receiving postoperative wholebreast radiotherapy; and (5) receiving systemic adjuvant therapy after surgery. ${ }^{3}$ Liu et al applied the criteria of the Z0011 trial to Chinese patients with sentinel lymph nodes positive breast cancer and screened outpatients with better prognosis and lower risk than those in the Z0011 study. ${ }^{4}$ These patients could safely only receive sentinel lymph node biopsy without axillary lymph node dissection. Systemic treatment, including chemotherapy, endocrine therapy, and immunotherapy, may remove residual tumor cells in the axilla and thus reduce the recurrence rate in the axilla. ${ }^{5}$ Studies have shown that $20-42 \%$ of patients with positive axillary lymph nodes can achieve complete pathological remission after receiving neoadjuvant therapy (chemotherapy or immunotherapy). ${ }^{6-8}$ The risk of local recurrence is also reduced. ${ }^{9}$ Adjuvant chemotherapy and endocrine therapy can also reduce the risk of regional recurrence. ${ }^{10,11}$ Despite this, $20-30 \%$ of patients with sentinel lymph node biopsy need to consider axillary lymph node dissection due to positive lymph nodes. ${ }^{12}$ The NSABP B04 test found that the recurrence rate of patients with insufficient axillary lymph node dissection was $18.6 \%{ }^{13}$ For elderly patients, ignoring axillary lymph node dissection will not affect DFS and OS. ${ }^{14,15}$ Axillary lymphadenectomy was associated with improved survival in patients presenting with clinical N2-3 invasive breast cancer. ${ }^{16}$ Most surgeons perform axillary lymph node dissection in patients with positive axillary lymph nodes. There are many reasons for this, such as difficulties in treating axillary recurrence, the psychological impact of recurrence in patients, and problems in a rigorous followup.

The effect of axillary therapy on survival is also controversial. For most patients with breast cancer, level III axillary lymph node dissection means an excessive treatment. ${ }^{17-19}$ Kodama et al compared the effects of $\mathrm{T} 1 /$
2/3 and N0/1a/1b (International Union of Cancer Staging 1987) breast cancer patients with level $\omega$ or level I axillary lymph node cleaning. ${ }^{17}$ The results show that the 10-year OS rates were $87.8 \%$ and $89.6 \%(P=0.552)$, and the 10 year disease-free survival rates were $74.1 \%$ and $76.6 \%$, respectively $(P=0.7)$. Because level III axillary lymph node cleaning did not improve the survival rate in $T 1 / 2 / 3$ and $\mathrm{N} 0 / 1 \mathrm{a} / 1 \mathrm{~b}$ patients, it is not recommended. Tominaga et al compared the effects on overall survival of level III with level II axillary lymph node cleaning in patients with stage II breast cancer. ${ }^{19}$ They found that the 10 -year overall survival rates were $86.6 \%$ and $85.7 \%(P=0.931)$, and the 10-year DFS rates were $73.3 \%$ and $77.8 \%$, respectively, in patients with level II or level III axillary lymph node dissection $(P=0.666)$. Therefore, they thought that extra level III axillary lymph node cleaning might be of no benefit. However, these two studies did not focus on patients with positive lymph nodes. The lymph node metastasis rates in Kodama and Tominaga's study were $32.1 \%$ and $32.7 \%$, respectively. In the study of Kodama, level III axillary lymph nodes invasion rate was only $7.4 \% .{ }^{17}$ Level III axillary lymph node dissection could remove a higher proportion of positive lymph nodes in lymph nodes positive breast cancer patients.

Although level III cleaning does not improve survival, ${ }^{20}$ metastasis of level III axillary lymph nodes is an essential factor that causes distant recurrence. ${ }^{21}$ It is an important index to judge patients' prognosis with breast cancer. Once tumor cells invade axillary lymph nodes, they will spread along with the lymphatic system. Consequently, tumor cells invading level III axillary lymph nodes are mostly to spread to the neck and the chest cavity. Level III axillary lymph node cleaning can increase the completeness of axillary lymph node cleaning in patients with preoperative positive axillary lymph nodes. It also has important guiding significance to postoperative treatment and prognosis improvement. Level III axillary lymph node invasion, however, is not an independent prognostic factor. Although level III axillary lymph node cleaning can provide more accurate classification according to the American Joint Committee on Cancer Staging, cleaning the axillary lymph node does not bring a significant survival benefit. ${ }^{21}$ Level I+II axillary lymph node dissection is standard, but level III cleaning is unusual. Only in selected patients with a wide range of axillary lymph node invasion and/or obvious level II/III axillary lymph node invasion, level III axillary lymph node dissection is performed. ${ }^{21}$ This situation mainly 
occurs in locally advanced breast cancer with clinical stages T3-4 and N1-2. ${ }^{22}$ Although level III axillary lymph node invasion is not an independent prognostic factor for regional recurrence, it may be a predictor for distant metastasis-free survival. Level III axillary lymph node dissection in modified radical mastectomy can reduce the risk of distant metastasis.

Early clinical studies of the extent of lymph node metastasis in positive lymph node breast cancer patients found that $20-58 \%$ of patients with axillary lymph node metastasis were restricted to level I; $20-29 \%$ and $16-32 \%$ of patients with lymph node metastasis were confined to levels I+II and levels I+III, respectively. About $20 \%$ of patients were diagnosed with pathological level III metastasis. $^{23,24}$ The level $\omega$ metastasis rate was highest in Khafagy's study. ${ }^{25}$ Of 59 positive axillary lymph node patients, $31(52.5 \%)$ had level III axillary lymph node metastasis. In a study by Tao et al, of 87 positive axillary lymph nodes patients, 18 (20.7\%) had level III axillary lymph node metastasis. ${ }^{26}$ Yildirim reported a level III axillary lymph node metastasis rate of $15-31 \% .^{21}$ In a study of T0-2 axillary lymph node-positive breast cancer patients who received neoadjuvant chemotherapy, $0.9 \%$ of patients had level III axillary lymph node invasion. ${ }^{27}$

Several studies have discussed the correlation between axillary lymph node metastasis and clinical-pathological factors. Toma et al reported that nuclear grade 3 was associated with level III axillary lymph node invasion. ${ }^{28}$ Ung et al found that it was related to axillary lymph node palpability, pathological tumor size, and lymph vessel invasion. ${ }^{29}$ Veronesi et al studied 539 patients with breast cancer and found that level III axillary lymph nodes were invaded in $16.9 \%$ of $\mathrm{T} 1$ breast cancer patients. ${ }^{24}$ This study also revealed the possibility that level II or III axillary lymph node invasion is connected with the number of metastatic nodes. In patients with only one level I axillary lymph node metastasis, 8\% also had higher levels of axillary lymph node metastasis; in patients with two -level I axillary lymph node metastases, $25.3 \%$ also had higher levels of axillary lymph nodes metastasis; and in patients with four or more level I axillary lymph node metastases, the rate was as high as $65.8 \%$. Yildirim found that level III axillary lymph node involvement, tumor size, lymph node, and vascular invasion, and the number of positive axillary lymph nodes were important pathological factors that predicted distant metastasis. ${ }^{21}$ In an original single-factor analysis, he found that tumor size, lymph node and vascular invasion, extracapsular involvement, and premenopausal status were associated with level III axillary lymph node metastasis; multiple-factor analysis showed that only the number of positive lymph nodes was related to level III axillary lymph node positivity, but this cannot be confirmed before surgery. In his study, the cut-off point of the number of positive nodes for level III involvement was 7. Dillon thought level III clearance could play a selective but definite role in patients with lymph node-positive breast carcinoma. ${ }^{12}$ Pathological features of primary tumors can help judge the risk of level III axillary lymph node invasion. In axillary lymph nodepositive breast cancer patients, level III infiltration predicting factors include: tumor size $(\mathrm{OR}=1.36$, 95\% CI: 1.2-1.5, $P<0.001)$, invasive lobular carcinoma $(\mathrm{OR}=$ 3.6, 95\% CI: 1.9-6.95, $P<0.001)$, extracapsular involvement $(\mathrm{OR}=0.27,95 \% \mathrm{CI}: 0.18-0.4, \mathrm{P}<0.001)$, and lymph node vascular infiltration $(\mathrm{OR}=0.58,95 \% \mathrm{CI}$ : $0.35-1, P=0.04)$. In patients with tumors larger than $3 \mathrm{~cm}$ in diameter, the risk of level III metastasis was as high as $34 \%$. In invasive lobular carcinoma patients with positive lymph nodes, the level III axillary lymph nodepositive rate was $41 \%$. Prediction factors of level III invasion in sentinel lymph node-positive patients included invasive lobular carcinoma $(\mathrm{OR}=4.1,95 \% \mathrm{CI}$ : $1-16.8$, $P=0.049)$, lymph node extracapsular involvement $(\mathrm{OR}=$ 0.18 , 95\% CI: $0.06-0.57, P=0.003)$, and at least one positive sentinel lymph node $(\mathrm{OR}=4.9,95 \% \mathrm{CI}: 1.5-16.1$, $P=0.009$ ). Fan's research showed that factors such as tumor size, lymph node biopsy methods, and primary tumor response to neoadjuvant chemotherapy by ultrasound could predict the risk of level III axillary lymph node involvement. ${ }^{27}$ The pathological complete response rate of axillary lymph nodes in the primary tumor effective group after neoadjuvant chemotherapy was significantly higher than that in the ineffective group $(36.5 \%$ [73/200] vs $19.1 \%$ [17/89], $P=0.003)$. Effective neoadjuvant chemotherapy might reduce the incidence rate of level III positivity. Node positivity as proved by ultrasoundguided needle biopsy, large tumor size, and primary tumor nonresponse to neoadjuvant chemotherapy are independent predictors of axillary lymph node positivity. The level III axillary lymph node-positive rate was only $4.9 \%$ in patients with no risk factors, and in patients with three risk factors ( $\mathrm{T}>2 \mathrm{~cm}$, axillary lymph node positivity as proved by fine needle biopsy and noneffective neoadjuvant chemotherapy), the rate of level III invasion was as high as $23.7 \%$. Patients who have two or more risk factors should be considered to have level III clearance because in these 
patients the rate of level $\amalg$ involvement was at least $20 \%$. Wang et al considered level III axillary lymph node dissection should be taken into account (i) for tumors at stage T3 or above whose estrogen receptor (ER) expression is negative as confirmed by preoperative breast tumor needle biopsy and (ii) for the intraoperative detection of axillary lymph node metastasis that is suspected to be of level I-II or to be accompanied by extra lymph node tissue infiltration. ${ }^{30}$ The number of axillary lymph node metastases at levels I-II, the involvement of external nodes, and the negative expression of ER are risk factors for axillary lymph node metastasis. Further stratified analysis showed that level III axillary lymph node metastasis was more likely to occur if the tumor above T3 was located laterally $(P=0.035)$. If ER expression is negative, with the increase of tumor stage, level III lymph nodes are more likely to metastasize.

Knowledge of the risk factors and the predictive factors of level III axillary lymph node metastasis can guide a suitable treatment strategy by the surgeon and/or tumor radiotherapist.

\section{Complications of Level III Axillary Lymph Node Dissection}

It has been reported that the dissection of level I-III axillary lymph nodes cannot improve the long-term efficacy of breast cancer surgery, ${ }^{13,31}$ but can increase the complications such as lymphedema of the affected limb, paresthesia, pain, limitation of shoulder joint activity, effusion, and so on, ${ }^{32}$ which may be caused by overtreatment. However, no significant axillary lymph node dissection complication has been found, and serious complications such as axillary vein/artery thrombosis or injury and axillary motor nerve injury have rarely been reported in the literature. Although level III axillary lymph node dissection takes longer and can result in more blood loss than the level I dissection, it does not affect its clinical effect. There were no statistically significant differences between the two groups in terms of follow-up symptoms such as upper limb pain, pectoralis major atrophy, upper limb motor function, and social function. ${ }^{19}$ The incidence of malformation associated with additional lymph node dissection did not increase in patients with breast cancer at similar stages who underwent standard axillary lymph node dissection. The incidence of postoperative upper extremity lymphedema ranges from $6 \%$ to $30 \%,{ }^{25}$ depending on the methods used to define lymphedema and follow-up adequacy. At present, it is generally believed that the incidence of lymphedema is very low if the adventitia of the axillary vein is not damaged. Therefore, surgeons should be cautious to avoid damaging the outer membrane around the axillary vein if they want to separate the brachial plexus fat above the axillary vein for lymph node dissection. ${ }^{33}$

\section{Surgical Options for Level III Axillary Lymph Node Dissection}

There are two commonly used ways to expose the subclavian area for level III dissection. One is to retract the pectoralis major and minor muscles. The other one is to split the pectoralis major muscle (Kodama method). On this basis, a series of studies put forward several improved ways. Muscolino et al proposed a practical method to reach level III axillary lymph nodes: splitting the pectoralis major muscle and immobilizing the pectoralis minor muscle. ${ }^{34}$ Although the exposed space is limited, this approach has been recommended by other clinicians. ${ }^{35}$ This method can expose the axillary apex and facilitate the dissection of the upper axillary vein lymph nodes (supraclavicular lymph nodes) that may be penetrated in locally advanced breast cancer. Also, prepectoral lymph node dissection is recommended because of muscle preservation. Alfredo et al found that subpectoral dissection usually results in retention of lymph nodes at the axillary apex. ${ }^{22}$ If we need to preserve the pectoralis major and minor muscles during dissection of level I-III lymph nodes in breast cancer patients, it is suggested to clean level III lymph nodes subpectoral through the posterior space of the pectoralis major muscle. Hadjiminas et al introduced a method conducive to safe cleaning of level III axillary lymph nodes. ${ }^{36}$ Access to level III is achieved through a muscle splitting transverse incision on the pectoralis major, centered on the point where the axillary vein crosses the first rib. This is located $5 \mathrm{~cm}$ lateral and $1 \mathrm{~cm}$ superior to the suprasternal notch. The pectoralis minor can be retracted laterally, and the neurovascular bundle to the pectoralis major can be dissected out of the surrounding fat and retracted in a silicone sling. It is recommended for patients undergoing mastectomy since the muscle splitting incision can be performed without the need for a separate skin incision. This method is especially ideal for patients with level III recurrence. It allows the surgeon to access level III without going through previously operated levels I and 
II, thereby minimizing the risk of nerve or vessel injury during dissection.

\section{Conclusion}

Underestimating the number of positive lymph nodes may lead to false assessments of the risk of distant metastasis, compromising local and regional control, which will have adverse effects on further treatment. ${ }^{25}$ It is essential to analyze the risk factors and the predictive factors of level III lymph node invasion to facilitate treatment strategy decisions. It is reasonable to consider dissection of level III axillary lymph nodes according to risk factors, but its effect on survival rate still needs further research.

\section{Disclosure}

The authors report no conflicts of interest in this work.

\section{References}

1. Jinno $\mathrm{H}$, Inokuchi $\mathrm{M}$, Ito $\mathrm{T}$, et al. The Japanese breast cancer society clinical practice guideline for surgical treatment of breast cancer, 2015 edition. Breast Cancer. 2016;23(3):367-377. doi:10.1007/ s12282-016-0671-x

2. Gradishar WJ, Anderson BO, Balassanian R, et al. NCCN guidelines insights: breast cancer, version 1.2017. J Natl Compr Canc Netw. 2017;15(4):433-451. doi:10.6004/jnccn.2017.0044

3. Giuliano AE, Hunt KK, Ballman KV, et al. Axillary dissection vs no axillary dissection in women with invasive breast cancer and sentinel node metastasis: a randomized clinical trial. JAMA. 2011;305 (6):569-575. doi:10.1001/jama.2011.90

4. Liu M, Wang S, Cui S, Duan X, Fan Z, Yu Z. The feasibility of the ACOSOG Z0011 criteria to chinese breast cancer patients: a multicenter study. Sci Rep. 2015;16(5):15241. doi:10.1038/ srep15241

5. Zhang L, Yu XL, Guo XM. Research progress of axillary management approach for 1-2 sentinel lymph node positive early stage breast cancer patients. Chin J Radiat Oncol. 2016;25(3):292-295.

6. Straver ME, Rutgers EJ, Russell NS, et al. Towards rational axillary treatment in relation to neoadjuvant therapy in breast cancer. Eur J Cancer. 2009;45(13):2284-2292. doi:10.1016/j.ejca.2009.04.029

7. Rouzier R, Extra JM, Klijanienko J, et al. Incidence and prognostic significance of complete axillary downstaging after primary chemotherapy in breast cancer patients with $\mathrm{T}_{1}$ to $\mathrm{T}_{3}$ tumors and cytologically proven axillary metastatic lymph nodes. J Clin Oncol. 2002;20(5):1304-1310. doi:10.1200/JCO.2002.20.5.1304

8. Alvarado R, Yi M, Le-petross $\mathrm{H}$, et al. The role for sentinel lymph node dissection after neoadjuvant chemotherapy in patients who present with node-positive breast cancer. Ann Surg Oncol. 2012;19 (10):3177-3184. doi:10.1245/s10434-012-2484-2

9. Mamounas EP, Anderson SJ, Dignam JJ, et al. Predictors of locoregional recurrence after neoadjuvant chemotherapy: results from combined analysis of national surgical adjuvant breast and bowel project B-18 and B-27. J Clin Oncol. 2012;30(32):3960-3966. doi:10.1200/JCO.2011.40.8369

10. Early Breast Cancer Trialists' Collaborative Group (EBCTCG). Effects of chemotherapy and hormonal therapy for early breast cancer on recurrence and 15-year survival: an overview of the randomised trials. Lancet. 2005;365(9472):1687-1717. doi:10.1016/S01406736(05)66544-0
11. Davies C, Godwin J. Early Breast Cancer Trialists'Collaborative Group (EBCTCG). Relevance of breast cancer hormone receptors and other factors to the efficacy of adjuvant tamoxifen: patient-level meta-analysis of randomised trials. Lancet. 2011;378(9793):771-784.

12. Dillon MF, Advani V, Masterson C, et al. The value of level III clearance in patients with axillary and sentinel node positive breast cancer. Ann Surg. 2009;249(5):834-839. doi:10.1097/SLA.0b01 3e3181a40821

13. Fisher B, Jeong JH, Anderson S, Bryant J, Fisher ER, Wolmark N. Twenty-five-year follow-up of a randomized trial comparing radical mastectomy, total mastectomy, and total mastectomy followed by irradiation. $N$ Engl J Med. 2002;347(8):567-575. doi:10.1056/ NEJMoa020128

14. Rudenstam CM, Zahrieh D, Forbes JF, et al. International Breast Cancer Study Group. Randomized trial comparing axillary clearance versus no axillary clearance in older patients with breast cancer: first results of international breast cancer study group trial 10-93. J Clin Oncol. 2006;24(3):337-344.

15. Martelli G, Boracchi P, De Palo M, et al. A randomized trial comparing axillary dissection to no axillary dissection in older patients with T1N0 breast cancer. Ann Surg. 2005;242(1):1-6. doi:10.1097/01. sla.0000167759.15670.14

16. Park TS, Thomas SM, Rosenberger LH, et al. The association between extension of axillary surgery and survival in women with N2-3 invasive breast cancer. Ann Surg Oncol. 2018;25 (10):3019-3029. doi:10.1245/s10434-018-6587-2

17. Kodama H, Nio Y, Iguchi C, Kan N. Ten-year follow-up results of a randomised controlled study comparing level-I vs level-III axillary lymph node dissection for primary breast cancer. $\mathrm{Br} J$ Cancer. 2006;95(7):811-816. doi:10.1038/sj.bjc.6603364

18. Kaufmann M, Morrow M, von Minckwitz G, Harris JR, Biedenkopf Expert Panel Members. Locoregional treatment of primary breast cancer: consensus recommendations from an international expert panel. Cancer. 2010;116(5):1184-1191. doi:10.1002/cncr.24874

19. Tominaga T, Takashima S, Danno M. Randomized clinical trial comparing level II and level III axillary node dissection in addition to mastectomy for breast cancer. Br J Surg. 2004;91(1):38-43. doi:10.1002/bjs.4372

20. Kong Y, Yang A, Xie X. Impact of the extent of axillary surgery in patients with $\mathrm{N} 2-3$ disease in the de-escalation era: a propensity score-matched study. Clin Transl Oncol. 2020. doi:10.1007/s12094020-02444-1

21. Yildirim E, Berberoglu U. Lymph node ratio is more valuable than level III involvement for prediction of outcome in node-positive breast carcinoma patients. World J Surg. 2007;31(2):276-289. doi:10.1007/s00268-006-0487-5

22. Barros AC, Andrade FE, Bevilacqua JL, et al. Radicality effect of adding an interpectoral to a subpectoral approach for dissection of level III axillary lymph nodes in breast cancer. Tumori. 2013;99 (4):500-504. doi:10.1177/030089161309900410

23. Rosen PP, Lesser ML, Kinne DW, Beattie EJ. Discontinuous or "skip" metastases in breast carcinoma. Analysis of 1228 axillary dissections. Ann Surg. 1983;197(3):276-283. doi:10.1097/ 00000658-198303000-00006

24. Veronesi U, Rilke F, Luini A, et al. Distribution of axillary node metastases by level of invasion: an analysis of 539 cases. Cancer. 1987;59(4):682-687. doi:10.1002/1097-0142(19870215)59:4<682:: AID-CNCR2820590403>3.0.CO;2-Z

25. Khafagy M, Mostafa A, Fakhr I. Distribution of axillary lymph node metastases in different levels and groups in breast cancer, a pathological study. J Egypt Natl Canc Inst. 2011;23(1):25-30. doi:10.1016/j.jnci.2011.07.004

26. Yangtao O, Jinfeng L, Tianfeng W, Lin BY. Exploration of the extent of axillary dissection for patients with node positive primary breast cancer. Chin J Surg. 2005;43(3):298-300. 
27. Fan Z, Li J, Wang T, et al. Level III axillary lymph nodes involvement in node positive breast cancer received neoadjuvant chemotherapy. Breast. 2013;22(6):1161-1165. doi:10.1016/j.breast.2013.08.004

28. Toma S, Leonessa F, Romanini A, et al. Predictive value of some clinical and pathological parameters on upper level axillary lymph node involvement in breast cancer. Anticancer Res. 1991;11(4):1439-1443.

29. Ung O, Tan M, Chua B, Barraclough B. Complete axillary dissection: a technique that still has relevance in contemporary management of breast cancer. ANZ J Surg. 2006;76(6):518-521. doi:10.1111/j.14452197.2006.03765.x

30. Wang R, Chen J, Tian CX, et al. High risk factors of the third level of lymphatic metastasis in breast cancer patients received radical modified mastectomy: an analysis of 747 cases. Chin J Surg. 2014;52(5):346-349.

31. Silverstein MJ, Skinner KA, Lomis TJ. Predicting axillary nodal positivity in 2282 patients with breast carcinoma. World J Surg. 2001;25(6):767-772. doi:10.1007/s00268-001-0003-x

32. Lucci A, McCall LM, Beitsch PD, et al., American College of Surgeons Oncology Group. Surgical complications associated with sentinel lymph node dissection (SLND) plus axillary lymph node dissection compared with slnd alone in the American college of surgeons oncology group trial z0011. J Clin Oncol. 2007;25 (24):3657-3663. doi:10.1200/JCO.2006.07.4062
33. Somers RG, Jablon LK, Kaplan MJ, Sandler GL, Rosenblatt NK. The use of closed suction drainage after lumpectomy and axillary node dissection for breast cancer. A prospective randomized trial. Ann Surg. 1992;215 (2):146-149. doi:10.1097/00000658-199202000-00009

34. Muscolino G, Leo E, Sacchini V, Bedini AV, Luini A. Resectable breast cancer: axillary dissection sparing pectoralis muscles and nerves. Eur J Surg Oncol. 1998;14:429-433.

35. Pai A, Gupta P, Raina S, Nadkarni MS, Parmar V, Badwe RA. Interpectoral approach to dissection of the axillary apex: an elegant and effective approach. J Surg Oncol. 2006;94(3):252-254.

36. Hadjiminas DJ, Zacharioudakis KE. Direct transpectoral approach for level III axillary lymph node clearance. Ann R Coll Surg Engl. 2014;96(6):481-482. doi:10.1308/rcsann.2014.96.6.481

\section{Publish your work in this journal}

Cancer Management and Research is an international, peer-reviewed open access journal focusing on cancer research and the optimal use of preventative and integrated treatment interventions to achieve improved outcomes, enhanced survival and quality of life for the cancer patient.
The manuscript management system is completely online and includes a very quick and fair peer-review system, which is all easy to use. Visit http://www.dovepress.com/testimonials.php to read real quotes from published authors. 